\title{
Adaptación y Validación en Español de la Self-Concealment Scale
}

\section{Adaptation and Validation of a Spanish-Language Version of the Self-Concealment Scale}

\author{
Catalina Letelier y Paula Errázuriz \\ Pontificia Universidad Católica de Chile
}

\begin{abstract}
Se presenta la adaptación al español y validación en una muestra chilena por conveniencia de la Escala de AutoOcultamiento (Self-Concealment Scale) de Larson y Chastain (1990). Participaron 193 adultos (100 hombres y 93 mujeres), con una edad media de 28,39 años $(D E=8,75)$, distribuidos en 3 grupos de la Región Metropolitana: 53 estudiantes universitarios, 64 estudiantes de un instituto de capacitación y 76 profesionales y dirigentes de una fundación sin fines de lucro. Para examinar la validez de la versión en español, se realizó un análisis factorial confirmatorio. Los resultados permiten inferir que la versión en español tiene propiedades psicométricas satisfactorias, presentando una alta consistencia interna $(\alpha=0,85)$ y una aceptable estabilidad a través del tiempo $(r=0,74)$. Al igual que la escala original, la versión en español validada resultó ser unidimensional y las puntuaciones de la escala se correlacionaron directamente con sintomatología depresiva y ansiosa, medida a través del Outcome Questionnaire 30.2 (OQ 30.2). Estos hallazgos avalan el uso de la versión en español de la EAO en población de habla hispana.
\end{abstract}

Palabras clave: escala de auto-ocultamiento, secreto, adaptación, validación, estudio metodológico

\begin{abstract}
This paper presents a Spanish-language adaptation of the Self-Concealment Scale (SCS) by Larson and Chastain (1990) and its validation in a Chilean convenience sample. The participants were 193 adults (100 men and 93 women) with a mean age of 28.39 years $(S D=8.75)$, grouped in 3 subsamples from the Metropolitan Region: 53 college students, 64 students from a technical institute, and 76 professionals and community leaders of a non-profit organization. In order to examine the validity of the Spanish-language version of the SCS, a confirmatory factor analysis was conducted. Results suggest that the Spanish-language version of the SCS has satisfactory psychometric properties, showing high internal consistency $(\alpha=0.85)$ and acceptable stability over time $(r=0.74)$. Like the original scale, the validated Spanish-language version proved to be unidimensional and its scores correlated directly with anxious and depressive symptomatology as measured with the Outcome Questionnaire 30.2 (OQ 30.2). These findings support the use of this version of the SCS with Spanish-speaking population.
\end{abstract}

Keywords: self-concealment scale, secret, adaptation, validation, methodological study

Para la psicología posmoderna subjetiva, el ser humano es intrínsecamente un contador de historias y es a través de estas que entiende el mundo (White \& Epston, 1990/2002). En la interacción social, las interpretaciones evolucionan y los nuevos hechos y sus consecuencias generan un cambio en la comprensión y evaluación que la persona tiene sobre sus historias individuales (Fivush, 2010). Narrar las experiencias requiere, por definición, hacer una selección y edición de la información, dejando fuera ciertos aspectos que son silenciados u ocultados. Es comprensible que aquello que se decide no pronunciar influya en las narraciones autobiográficas y tenga implicancias en el actuar del sujeto y en la generación de sentido acerca de lo que lo rodea. Es más, mantener algo oculto podría generar narrativas alternativas que no se ajustan al guión construido, implicando cambiar la manera de entender las narraciones anteriores (Fivush, 2010).

La mayoría de las personas admiten que de manera consciente ocultan a otros información acerca de sí mismas (Cramer \& Barry, 1999), incluso cuando revelar información puede ir en su propio beneficio. Por ejemplo, la mitad de los pacientes en psicoterapia mantiene secretos de sus terapeutas (Farber, Berano \&

Catalina Letelier y Paula Errázuriz, Escuela de Psicología, Pontificia Universidad Católica de Chile, Santiago, Chile.

El artículo es parte de la tesis de la primera autora para optar al Grado de Magíster en Psicología Clínica en la Pontificia Universidad Católica de Chile. Se agradece la colaboración del Dr. Patricio Cumsille por su valiosa contribución al manuscrito; y al Dr. Dale G. Larson por su apoyo a lo largo de esta investigación.

Este estudio recibió apoyo económico del Fondo de Innovación para la Competitividad del Ministerio de Economía, Fomento y Turismo de Chile, a través de la Iniciativa Científica Milenio, Proyecto IS130005, y de la Comisión Nacional de Investigación Científica y Tecnológica de Chile, a través de los proyectos FONDECYT 11110041 y 1191299.

La correspondencia relativa a este artículo debe ser dirigida a Paula Errázuriz Arellano, Escuela de Psicología, Pontificia Universidad Católica de Chile, Avda. Vicuña Mackenna 4860, Macul, Santiago, Chile. E-mail: paulae@uc.cl 
Capobianco, 2004), aun cuando ocultar información en este contexto se considera perjudicial para el proceso (Cepeda-Benito \& Short, 1998). Diversas investigaciones han demostrado que el ocultamiento ocurre en distintos países y en una amplia pluralidad de temáticas, desde triviales hasta experiencias traumáticas (Wismeijer, 2011), tales como bienestar subjetivo (Masuda et al., 2011), depresión (Laird, Marrero, Melching \& Kuhn, 2013), abuso sexual (Dorahy \& Clearwater, 2012), autoestima (Zhang, Wang, Li, Yu \& Bi, 2011), homosexualidad (Schrimshaw, Siegel, Downing Jr. \& Parsons, 2013), VIH (Maas, Wismeijer, Van Assen \& Aquarius, 2012) y empleabilidad (Wheat, Brohan, Henderson \& Thornicroft, 2010).

El auto-ocultamiento es un tipo específico de ocultamiento. Larson y Chastain (1990) lo definen como una predisposición a ocultar activamente información personal a otros que es percibida por el sujeto como negativa o estresante. La primera parte de esta definición (predisposición a ocultar activamente) supone que la información auto-ocultada es accesible a la consciencia y deliberadamente velada por un esfuerzo mental y conductual. Este componente de inhibición conductual del auto-ocultamiento deja fuera el contenido disociado, escindido, negado u olvidado (Larson \& Chastain, 1990). La segunda parte de esta definición (información personal percibida por el sujeto como negativa o estresante) implica que la información ocultada es acerca de sí mismo, catalogada como altamente íntima, personal y negativa. El miedo asociado a la revelación de dicha información la hace estresante o angustiosa (Larson \& Chastain, 1990), aludiendo a preocupaciones, secretos, convicciones de inadecuación y arrepentimientos que se refieren a uno mismo y no tanto a las ofensas y las violaciones de reglas (Wismeijer, 2011).

Se ha encontrado que el auto-ocultamiento se correlaciona con el bienestar, tanto físico como psicológico, de las personas y con los efectos en los resultados psicoterapéuticos. Un mayor autoocultamiento se acompaña de mayor psicopatología, enfermedades fisiológicas, altos niveles de angustia, baja autoestima, mayores conflictos interpersonales, redes de apoyo social disminuidas y mayor dificultad en obtener resultados positivos en los procesos psicológicos (Cepeda-Benito \& Short, 1998).

La mayor parte de las investigaciones (e.g., Cepeda-Benito \& Short, 1998; Masuda et al., 2011; Uysal, Lee Lin \& Knee, 2010) muestran que ocultar información se asocia a consecuencias dañinas para la salud, que pueden llegar a ser, incluso, peores que el evento inicial escondido (e.g., Wismeijer, Van Assen, Sijtsma \& Vingerhoets, 2009). Las consecuencias personales negativas de ocultar información incluyen problemas físicos (e.g., dolores de cabeza o nauseas), psicológicos (e.g., ansiedad o depresión) y sociales (e.g., baja autoestima, mayores conflictos interpersonales y redes de apoyo social disminuidas). Por ejemplo, los hombres homosexuales que ocultan su orientación sexual presentan mayor riesgo de padecer cáncer o alguna enfermedad infecciosa (Kelly, 1999) y el mantenimiento de secretos de los adolescentes hacia sus padres se asocia con depresión y conducta antisocial (Laird et al., 2013).

Las personas mantienen secretos por miedo real o imaginado a las repercusiones de la exposición, en particular por el temor a ser estigmatizado y volverse indeseables, ya que se cree que la revelación puede afectar la imagen social y personal (Kelly, 1999; Wegner \& Lane, 1995). Una de las teorías que explica los efectos negativos del auto-ocultamiento es el aumento de trabajo fisiológico resultante de la inhibición del comportamiento que acompaña el ocultar conscientemente información a otros, aumentando, a su vez, el estrés, lo que se sabe que se asocia a mayores problemas mentales y físicos (Cramer \& Barry, 1999; Pennebaker \& O'Heeron, 1984).

Aunque las posibles ventajas del auto-ocultamiento han sido estudiadas con menor frecuencia, existen algunos estudios que sugieren que auto-ocultar no siempre conlleva consecuencias negativas, pudiendo ser defensivo, adaptativo o protector del ego (Pennebaker, Colder \& Sharp, 1990). Por ejemplo, el auto-ocultar síntomas y conductas de una enfermedad mental protege a las personas del estigma social (Corrigan \& Rao, 2012).

En esta línea, Wegner y Lane (1995) proponen que revelar o interrumpir el acto de mantener secretos es el primer paso hacia tratamientos exitosos para muchos trastornos, como disminuir el riesgo de suicidio (Kelly \& Yip, 2006), disminuir la sintomatología del VIH o la del trastorno por estrés post-traumático (Ironson et al., 2013), mejorar el estado de ánimo u optimizar el funcionamiento físico de enfermos de artritis reumatoide (Kelley, Lumley \& Leisen, 1997). Sin embargo, la revelación no siempre presenta efectos positivos. Estudios referidos al abuso sexual muestran que muchas veces revelar el abuso genera conductas de desconfianza e incredulidad por parte de terapeutas y cercanos del revelador, causando una protección deficiente, negación y estigmatización, pudiendo ser más dañina la revelación que el secreto mismo (Dorahy \& Clearwater, 2012). 
Existe un debate acerca de cuál es el efecto que tiene el auto-ocultamiento en la probabilidad de que una persona consulte a un psicoterapeuta. Por un lado, Kelly y Achter (1995) muestran que aquellas personas que puntúan alto en su nivel de auto-ocultamiento tienen un $50 \%$ más de probabilidades de consultar a un terapeuta, dado que mantienen menores redes sociales de apoyo, por lo que el espacio terapéutico les resulta un lugar apropiado para poder compartir sus secretos más íntimos. Por otro lado, Cepeda-Benito y Short (1998) encuentran que lo que influye en la búsqueda de ayuda terapéutica no es el nivel de auto-ocultamiento de la persona, sino que la presencia de angustia. En una tercera postura, Cramer y Barry (1999) muestran que la relación entre búsqueda de ayuda psicológica y auto-ocultamiento es de carácter indirecto, ya que las personas son más propensas a buscar ayuda terapéutica cuando sus niveles de estrés están altos, los cuales aumentan considerablemente cuando ocultan información personal a otros.

Del mismo modo, se discute cómo influye el auto-ocultamiento en el desarrollo de la psicoterapia. Como consecuencias negativas se observó que quienes tienen un alto nivel de auto-ocultamiento mantienen actitudes menos favorables durante la terapia, probablemente porque temen que esta les obligará a revelar sus pensamientos más íntimos (Cramer \& Barry, 1999; Kelly \& Achter, 1995). Las consecuencias positivas del auto-ocultamiento durante la psicoterapia incluyen: mantener el equilibrio de poder en la diada paciente-terapeuta, acotar los problemas a trabajar y ser una defensa cuando el paciente no está listo para revelar información angustiante (Hill, Gelso \& Mohr, 2000).

Para determinar la tendencia de una persona a auto-ocultar, Larson y Chastain (1990) desarrollaron la escala Self-Concealment Scale (SCS) o Escala de Auto-Ocultamiento (EAO), siendo este el único instrumento que mide el concepto de auto-ocultamiento sin otros fenómenos concurrentes. Existen otros instrumentos que miden autorrevelación en vez de auto-ocultamiento: el Self-Disclosure Index (SDI; Miller, Berg \& Archer, 1983) y el Distress Disclosure Index (DDI; Kahn \& Hessling, 2001). El SDI es una escala de autoadministración de 11 ítems, que mide la disposición de una persona a revelar en el futuro información personal a un extraño del mismo sexo, enfatizando en su medición la revelación de información general. El DDI es una escala autoadministrada de 10 ítems, que mide la tendencia de una persona a revelar información personalmente angustiante.

A pesar de la relevancia del auto-ocultamiento y de que un 5,68\% de la población mundial habla español como primera lengua (Moreno Fernández \& Otero Roth, 2006), hasta la fecha la revisión realizada no ha encontrado publicaciones que den cuenta de la adaptación y validación al español de la EAO. En contraste, en Turquía Deniz y Çok (2010) realizaron una adaptación y validación de esta al turco, obteniendo una consistencia interna similar a la de la escala original ( $\alpha$ de Cronbach $=0,86$ ) y confirmando su estructura unifactorial.

En el presente estudio se optó por adaptar y validar la EAO al español, debido a que es un instrumento de calidad que presenta buenas propiedades psicométricas, tanto en su validación inicial como en revisiones y adaptaciones posteriores (Cramer \& Barry, 1999; Deniz \& Çok, 2010; Larson \& Chastain, 1990). A su vez, ha sido utilizada en población con un amplio rango de edad, tanto en adultos como adolescentes (e.g., Cepeda-Benito \& Short, 1998; Laird et al., 2013), en variadas culturas, como la estadounidense (e.g., Wismeijer, 2011), turca (e.g., Deniz \& Çok, 2010), inglesa (Cruddas, Gilbert \& McEwan, 2012) y coreana (Nam et al., 2013), y en diferentes contextos sociales e interpersonales (Masuda et al., 2011).

Si bien el diseño de la escala original y sus revisiones y adaptaciones fueron realizadas principalmente durante la década de los 90, ha habido una considerable cantidad de literatura que se ha enfocado en el constructo de auto-ocultamiento, utilizando la SCS, durante las décadas posteriores a su creación (e.g., Cruddas et al., 2012; Laird et al., 2013; Nam et al., 2013). Recientemente, los autores de la escala original realizaron una revisión de 137 estudios empíricos que se han enfocado en el constructo de autoocultamiento utilizando la SCS (Larson, Chastain, Hoyt \& Ayzenberg, 2015). Adicionalmente, diseñaron un sitio web denominado Self-Concealment Research Collaborative, con la finalidad de aunar la creciente cantidad de investigación en auto-ocultamiento y los fenómenos asociados (Larson, s.f.).

El uso de la EAO en contextos clínicos y en investigación puede ser provechoso, porque es una escala de autoaplicación que solo requiere de las instrucciones impresas y es fácil y rápida de administrar y codificar. Además de ser una escala factible de incluir en investigaciones debido a su bajo costo y sencillez, la EAO puede ser un aporte en la práctica en psicoterapia individual, de parejas y de grupo, trabajo con víctimas de abuso sexual y maltrato, psicología forense y práctica clínica en general. 


\section{Desarrollo de la Escala de Auto-Ocultamiento}

La EAO es una escala breve de autoaplicación con altos niveles de confiabilidad reportados en términos de consistencia interna (a de Cronbach $=0,83)$ y test-retest $(r=0,81)$ luego de cuatro semanas (Larson \& Chastain, 1990). En el estudio original participaron 29 hombres y 277 mujeres de entre 21 y 79 años $(M=$ 42 años, $D E=10,9$ ), de los cuales $82 \%$ tenía estudios universitarios. Al someter la escala a un análisis factorial exploratorio, se determinó la unidimensionalidad de la escala, con un factor que mide la tendencia a mantener secretos angustiantes.

La escala se correlacionó directamente con síntomas físicos, depresión, ansiedad y baja frecuencia de contar a los demás un secreto muy personal, y se correlacionó inversamente con autorrevelación, soporte social y red social compuesta por amigos (Larson \& Chastain, 1990).

La unidimensionalidad de la EAO fue apoyada en estudios posteriores realizados en diferentes países. Por ejemplo, dos estudios diferentes que utilizaron análisis factorial confirmatorio con estudiantes canadienses (Cramer \& Barry, 1999) y turcos (Deniz \& Çok, 2010), y otro estudio que utilizó análisis de componentes principales y el modelo de análisis de escalas de Mokken (Mokken \& Lewis, 1982) en una muestra representativa de población holandesa (Wismeijer, Sijtsma, Van Assen \& Vingerhoets, 2008) apoyaron la unidimensionalidad de la escala. Estas investigaciones también obtuvieron valores altos en las estimaciones de la consistencia interna $(\alpha=0,86)$ y confiabilidad test-retest $(r=0,74)$.

Considerando la importancia del auto-ocultamiento y la falta de instrumentos para medirlo en la población hispanohablante, la presente investigación tuvo por objetivo realizar una adaptación al español y una revisión de las propiedades psicométricas de la EAO (Larson \& Chastain, 1990). Específicamente, se propuso evaluar la confiabilidad y validez de la adaptación en una muestra de habla hispana. Un propósito fundamental de esta investigación psicométrica fue la contrastación empírica de la noción de autoocultamiento en población hispanohablante.

\section{Método}

\section{Participantes}

La muestra estuvo compuesta por 193 personas adultas, 93 (48,18\%) mujeres y 100 (51,81\%) hombres, entre 18 y 60 años $(M=28,39, D E=8,75)$. En relación a su nivel educacional, el 8,29\% tenía estudios básicos completos, el 45,59\%, enseñanza media completa, el 5,69\%, estudios técnicos completos, el 35,75\% era profesional y el 4,66\% tenía estudios de postítulo. Respecto del ingreso familiar mensual, el $39,89 \%$ de los participantes reportó un ingreso menor a $\$ 500.000$ pesos chilenos (aproximadamente US\$ 650), 26,94\% entre $\$ 500.000$ y $\$ 1.250 .000$ (aproximadamente entre US\$ 650 y US $\$ 1.650$ ), $15,54 \%$ entre $\$ 1.250 .000$ y $\$ 2.000 .000$ (aproximadamente entre US $\$ 1.650$ y US $\$ 2650$ ) y $17,61 \%$ mayor a $\$ 2.000 .000$ (aproximadamente US\$ 2.650), siendo esta la categoría de mayor ingreso. Para una mejor evaluación de estos datos es posible tomar como indicador de referencia el valor de ingreso mínimo mensual en Chile, que es de $\$ 270.000$ (aproximadamente US\$ 355). Un 4,66\% de los participantes pertenecían a una minoría étnica.

La muestra se recolectó por conveniencia en la Región Metropolitana de Chile e incluyó tres grupos, con el fin de que fuera diversa en cuánto a nivel socioeconómico, edad y sexo. El primer grupo se conformó con 53 estudiantes universitarios de variados estratos sociales, de los cuales 19 eran hombres y 34 mujeres, con un rango de edad entre 18 y 27 años $(M=20,85, D E=1,84)$. El segundo grupo incluyó a 64 estudiantes de un instituto de capacitación pertenecientes al quintil de ingreso más bajo de Chile, de los cuales 40 eran hombres y 24 mujeres, con un rango de edad entre 21 y 60 años $(M=35,88, D E=9,70)$. Por último, el tercer grupo incluyó a 76 profesionales y dirigentes sociales de una fundación sin fines de lucro, de los cuales 41 eran hombres y 35 mujeres, con un rango de edad entre 21 y 48 años $(M=27,36, D E=5,07)$.

\section{Instrumentos}

Escala de Auto-Ocultamiento (EAO). Esta escala autoadministrada incluye 10 ítems tipo Likert (verlos en la Tabla 1), con cinco alternativas de respuestas graduadas desde $1=$ muy en desacuerdo a $5=$ muy de acuerdo, que miden la tendencia de una persona a ocultar información personal que le resulta estresante o negativa. Produce un puntaje con un rango que va de los 10 a los 50 puntos, siendo los puntajes más altos indicativos de un mayor nivel de auto-ocultamiento. La consistencia interna de la escala 
ha sido estimada, mediante el coeficiente alfa de Cronbach, en 0,83 (Larson \& Chastain, 1990), en tanto la confiabilidad test-retest ha sido estimada en $r=0,81$ (Larson \& Chastain, 1990). El tiempo empleado en responder la escala es de 5 a 10 minutos.

Outcome Questionnaire (OQ-30.2). Este cuestionario autoadministrado es una versión abreviada del $\mathrm{OQ}$ 45.2. El instrumento se utiliza para evaluar el progreso en psicoterapia, evaluando tres dimensiones relacionadas con el bienestar psicológico (confort subjetivo, relaciones interpersonales y desempeño del rol social). Incluye 30 ítems tipo Likert (e.g., Nada me interesa, Estoy satisfecho con mi vida), con cinco alternativas de respuestas graduadas desde $0=$ nunca a $4=$ casi siempre, que miden el estado actual sintomatológico de una persona. El rango de puntajes posible va de 0 a 120 puntos, siendo los puntajes más altos indicativos de mayor presencia de sintomatología angustiosa — principalmente ansiedad, depresión, problemas somáticos y estrés-, y dificultades interpersonales, en el rol social y en la calidad de vida. Puntajes menores a 44 puntos sugieren que la persona no está más perturbada que la población general. El instrumento ha sido adaptado y validado en Chile (Errázuriz, Opazo, Behn, Silva \& Gloger, 2017), presentando apropiadas propiedades psicométricas. La consistencia interna del OQ 30.2 ha sido estimada mediante alfa de Cronbach en 0,88 y su confiabilidad test-retest en $r=0,82$ (Errázuriz et al., 2017).

Cuestionario de Antecedentes. Cuestionario de autoaplicación de 13 preguntas que registra características sociodemográficas, incluyendo: sexo, edad, nacionalidad, ingreso familiar mensual, nivel educacional, situación laboral, estado civil, creencia religiosa y experiencia anterior o actual en psicoterapia o psiquiatría.

\section{Procedimiento}

Se solicitó a los autores originales de la SCS un permiso firmado para la adaptación y validación de la EAO y se firmó un acuerdo de propiedad intelectual, siguiendo el proceder recomendado por la International Test Commission.

Un psicólogo bilingüe, cuya lengua materna es el español, realizó una traducción de la EAO del inglés al español. Un segundo psicólogo bilingüe, cuya lengua materna es el inglés, retradujo esta versión en español al inglés. Un tercer psicólogo bilingüe, cuya lengua materna es el español, comparó la versión traducida y la retraducida. Luego, un panel de expertos revisó la versión en español con el fin de evaluar la aplicabilidad a la población, la adecuada traducción de cada ítem y que el fenómeno medido se diera en Chile, conociendo desde un principio el objetivo del estudio. El panel de expertos estuvo compuesto por cuatro psicólogas académicas con diferentes formaciones teóricas y prácticas, que revisaron de manera independiente los ítems propuestos. Ninguna de ellas era parte del equipo de investigación. Las expertas concordaron en que el auto-ocultamiento es un fenómeno que acontece en Chile y que ocurre en una amplia pluralidad de temáticas de la sociedad. El panel concordó en que no había problemas en cuanto a la administración del cuestionario a una población de habla hispana, la comprensión de las opciones de respuesta ni la longitud del instrumento. El cuestionario resultó fácil y atractivo. A partir de sus observaciones se conservaron todos los ítems.

Una vez concluida esta fase, se invitó a los diferentes grupos muestrales a participar en el estudio, a los alumnos universitarios se les convidó al final de clases, a los del instituto de capacitación, al comienzo y a los de la fundación, durante su horario laboral. La participación fue confidencial y voluntaria, sin ningún tipo de compensación por participar en el estudio. Previo a la administración se le informó a la persona qué implicaba su participación y se explicitó su posibilidad de retirarse en cualquier momento sin perjuicio alguno. Quienes aceptaron participar firmaron un consentimiento informado.

Todos los instrumentos, que no requieren de capacitación previa para ser aplicados, fueron administrados en el mismo orden en el que se describieron y por la misma persona. El tiempo de aplicación total de la EAO, el OQ-30,2 y el Cuestionario de Antecedentes fue alrededor de 25 minutos. Los alumnos universitarios y del instituto de capacitación completaron los cuestionarios en la sala de clases, mientras los de la fundación, en su escritorio de trabajo.

De la muestra total fueron recontactados 49 participantes, 10 universitarios y 39 de la fundación, a quienes se les aplicó en una segunda ocasión la EAO, con cinco semanas de diferencia entre ambas mediciones. En ambas aplicaciones ningún ítem dejó de ser respondido por un número significativo de participantes. 


\section{Plan de Análisis}

Luego de revisar los descriptivos de los ítems (Tabla 1), se realizó un análisis factorial confirmatorio (AFC) para evaluar la unidimensionalidad del instrumento reportada en estudios anteriores (Cramer \& Barry, 1999; Deniz \& Çok, 2010; Larson \& Chastain, 1990; Wismeijer et al., 2008). El AFC se realizó utilizando el estimador de mínimos cuadrados ponderados diagonalizados (diagonally weighted least squares [DWLS]) para datos ordinales con errores estándar robustos (Rosseel, 2012). Como medidas de ajuste se utilizaron diferentes indicadores, usando los criterios de corte sugeridos en la literatura (Hu \& Bentler, 1999, Kline, 2016): Índice comparativo de Bentler-Bonett (CFI, > 0,95), Índice de Tucker-Lewis (TLI, $\geq 0,95$ ), Raíz del error cuadrático medio de aproximación (RMSEA, $\leq 0,08$ ) y Raíz del residuo cuadrático medio estandarizado (SRMR, $\leq 0,08$ ). Se utilizó el estimador de mínimos cuadrados ponderados con media y varianza ajustada (WLSMV), por ser más robusto a la no-normalidad de ítems ordinales, y se reportan las versiones robustas de todos los índices de ajuste.

La confiabilidad de la escala se estimó utilizando el coeficiente alfa de Cronbach, como medida de la consistencia interna de la escala total, y la estabilidad temporal de los puntajes obtenidos en la escala, a través de la correlación lineal de Pearson test-retest. Para evaluar la capacidad de discriminación de los ítems se obtuvieron los coeficientes de correlación ítem-test (Morales Vallejo, 2008).

Se compararon los promedios de la escala por categoría muestral y sexo, mediante la prueba $t$ de Student, y por categoría de edad, a través de análisis de varianza. Finalmente, el peso relativo de cada variable en la predicción del puntaje total de la EAO se estimó a través de una regresión lineal múltiple.

Para medir la validez referida a un criterio externo de la escala se correlacionó esta ( $r$ lineal de Pearson) con los resultados obtenidos en el OQ-30.2. Dicha elección se justificó en que Larson y Chastain (1990) encontraron correlaciones directas entre el auto-ocultamiento y medidas de depresión y ansiedad, sintomatología que es medida en el OQ 30,2 (Lambert, Finch, Okiishi \& Burlingame, 2005). Se esperaba obtener una correlación similar a la de la escala original con la presencia de síntomas depresivos $(r=0,41)$ y ansiosos $(r=0,32)$, es decir, a mayor tendencia a auto-ocultar mayor presencia de sintomatología.

Para manejar los datos perdidos en todos los análisis, excepto para el AFC, en que se utilizó el método de máxima verosimilitud con información completa (full information maximum likelihood; Rosseel, 2012), se realizó una imputación por expectación-maximización para el 0,41\% de los datos de la EAO y 1,79\% de los datos del Cuestionario de Antecedentes. Para el OQ 30.2, que correspondían al 0,69\% de los datos, se completaron según las indicaciones del manual de asignación de puntajes, reemplazándolos por el número entero más cercano al promedio del puntaje total de la persona (Lambert et al., 2005). No fue necesario imputar los datos de la segunda aplicación de la EAO, ya que no hubo datos perdidos. No se detectaron valores atípicos con puntajes mayores que \pm 3,29 (Tabachnick \& Fidell, 2001).

Los análisis de datos fueron realizados en el paquete estadístico SPSS 20 (Bühl, 2011), excepto el AFC, para el que se utilizó la librería Lavaan de R (R Core Team, 2017; Rosseel, 2012).

\section{Resultados}

En la Tabla 1 se puede observar que el puntaje total de la escala muestra un promedio de 25,78 puntos $(D E=8,58)$. Para todos los ítems el rango de puntaje obtenido fue equivalente al puntaje mínimo y máximo posible, en tanto el puntaje total máximo alcanzó a 49 puntos, un punto menos que el valor máximo posible.

\section{Análisis Factorial Confirmatorio}

En la Tabla 2 se presenta la matriz de intercorrelaciones entre los ítems de la escala. Se puede observar que todas las correlaciones son directas y con un rango de valores entre 0,22 y 0,59 , considerados pequeños a moderados, lo que indica que los ítems están relacionados entre sí, pero, a su vez, discriminan y no son redundantes (Pardo Merino \& Ruiz Díaz, 2002). Como dichas correlaciones resultan ser directas, es esperable que aquellas personas que puntúan alto en uno de los ítems también lo hagan en los demás.

El AFC con un solo factor (ver las cargas en la Tabla 3) mostró un modelo que presentó un ajuste moderado de los datos: $\chi^{2}(35, N=188)=231,056, p<0,001, \mathrm{CFI}=0,965$, TLI $=0,955$, RMSEA $=0,173$, $\mathrm{SRMR}=0,082$. La inspección de los índices de modificación sugirió la existencia de una correlación residual entre los ítems 3 y 5 , lo que se consideró apropiado, dado que ambos ítems se refieren a guardar secretos 
(utilizan explícitamente el verbo guardar), por lo que pueden compartir varianza. Esta nueva especificación del modelo mejoró sustancialmente el ajuste del modelo a los datos: $\chi^{2}(34, N=188)=156,863, p<0,001$, $\mathrm{CFI}=0,959$, TLI $=0,946, \mathrm{RMSEA}=0,100, \mathrm{SRMR}=0,069$.

Tabla 1

Descriptivos de los Ítems y el Puntaje Total de la EAO

\begin{tabular}{|c|c|c|c|c|}
\hline Ítem & Promedio & $\begin{array}{l}\text { Desviación } \\
\text { estándar }\end{array}$ & $\begin{array}{l}\text { Puntaje } \\
\text { mínimo }\end{array}$ & $\begin{array}{l}\text { Puntaje } \\
\text { máximo }\end{array}$ \\
\hline 1. Tengo un secreto importante que no he compartido con nadie & 2,82 & 1,36 & 1 & 5 \\
\hline $\begin{array}{l}\text { 2. Si compartiera todos mis secretos con mis amigos, me } \\
\text { apreciarían menos }\end{array}$ & 2,15 & 1,15 & 1 & 5 \\
\hline 3. Hay muchas cosas sobre mí que me guardo para mí mismo & 3,32 & 1,36 & 1 & 5 \\
\hline 4. Algunos de mis secretos me han atormentado bastante & 2,53 & 1,35 & 1 & 5 \\
\hline 5. Cuando algo malo me pasa, tiendo a guardármelo & 2,96 & 1,33 & 1 & 5 \\
\hline 6. A menudo me asusta revelar información sin querer & 2,65 & 1,27 & 1 & 5 \\
\hline $\begin{array}{l}\text { 7. Contar un secreto generalmente se vuelve en mi contra, por lo } \\
\text { que desearía no haberlo contado }\end{array}$ & 2,46 & 1,32 & 1 & 5 \\
\hline $\begin{array}{l}\text { 8. Tengo un secreto que es tan privado que mentiría si alguien me } \\
\text { preguntara sobre él }\end{array}$ & 2,55 & 1,37 & 1 & 5 \\
\hline $\begin{array}{l}\text { 9. Mis secretos son demasiado vergonzosos para compartirlos con } \\
\text { otros }\end{array}$ & 2,08 & 1,10 & 1 & 5 \\
\hline $\begin{array}{l}\text { 10. Tengo pensamientos negativos sobre mí mismo que nunca } \\
\text { comparto con nadie }\end{array}$ & 2,39 & 1,30 & 1 & 5 \\
\hline Total & 25,78 & 8,58 & 10 & 49 \\
\hline
\end{tabular}

Tabla 2

Correlaciones entre los Ítems de la EAO

\begin{tabular}{ccccccccccc}
\hline Item & 1 & 2 & 3 & 4 & 5 & 6 & 7 & 8 & 9 & 10 \\
\hline 1 & 1 & & & & & & & & & \\
2 & $0,29^{*}$ & 1 & & & & & & & & \\
3 & $0,47^{*}$ & $0,30^{*}$ & 1 & & & & & & & \\
4 & $0,59^{*}$ & $0,27^{*}$ & $0,43^{*}$ & 1 & & & & & & \\
5 & $0,38^{*}$ & $0,22^{*}$ & $0,58^{*}$ & $0,37^{*}$ & 1 & & & & & \\
6 & $0,36^{*}$ & $0,35^{*}$ & $0,37^{*}$ & $0,43^{*}$ & $0,39^{*}$ & 1 & & & & \\
7 & $0,39^{*}$ & $0,36^{*}$ & $0,34^{*}$ & $0,38^{*}$ & $0,34^{*}$ & $0,57^{*}$ & 1 & & & \\
8 & $0,48^{*}$ & $0,28^{*}$ & $0,31^{*}$ & $0,41^{*}$ & $0,20^{*}$ & $0,33^{*}$ & $0,47^{*}$ & 1 & & \\
9 & $0,40^{*}$ & $0,41^{*}$ & $0,26^{*}$ & $0,41^{*}$ & $0,30^{*}$ & $0,28^{*}$ & $0,40^{*}$ & $0,55^{*}$ & 1 & \\
10 & $0,30^{*}$ & $0,34^{*}$ & $0,28^{*}$ & $0,35^{*}$ & $0,30^{*}$ & $0,30^{*}$ & $0,36^{*}$ & $0,35^{*}$ & $0,41^{*}$ & 1 \\
\hline$* p<0,01 . N=193$ & & & & & & & & &
\end{tabular}

\section{Confiabilidad}

El coeficiente alfa de Cronbach estimado para la EAO muestra una consistencia interna similar a la de la escala original, $a=0,85$ (Larson \& Chastain, 1990) y a los estudios posteriores (e.g., Cramer \& Barry, 1999; Deniz \& Çok, 2010). En todos los ítems los coeficientes de correlación ítem-test resultaron altos, con un rango de valores entre 0,46 y 0,62 , mostrando una buena discriminación. Si se elimina un elemento, los valores alfa fueron aceptables y similares entre ellos, con un rango entre 0,83 y 0,85. Esto demuestra que la eliminación de cualquiera de ellos no produciría un aumento significativo en la consistencia interna de la escala, por lo cual se mantuvo la totalidad de los ítems sujetos a análisis. 
Tabla 3

Matriz de Cargas Factoriales del

Modelo Unidimensional de la $E A O$

\begin{tabular}{cc}
\hline Ítem & Carga factorial \\
\hline 1 & 0,742 \\
2 & 0,582 \\
3 & 0,595 \\
4 & 0,741 \\
5 & 0,532 \\
6 & 0,682 \\
7 & 0,735 \\
8 & 0,715 \\
9 & 0,755 \\
10 & 0,588 \\
\hline
\end{tabular}

$N=193$

El coeficiente de confiabilidad test-retest obtenido fue de $r=0,74(p<0,001)$, el cual resulta ser aceptable y similar al hallado en la escala original (Larson \& Chastain, 1990) y su revisión posterior (Cramer \& Barry, 1999), lo que indica una buena estabilidad de los puntajes en el tiempo. Al comparar, mediante $t$ para grupos dependientes, el promedio de los puntajes de la primera aplicación $(M=24,12, D E=$ $7,21)$ con los de la segunda aplicación $(M=23,88, D E=7,85)$, se observa que no difieren, $t(48)=0,31, p=$ 0,621 , lo que refuerza la estabilidad de este instrumento.

\section{Validez Referida a un Criterio Externo}

Al predecir el nivel de auto-ocultamiento según la presencia de sintomatología medida con el OQ 30.2, controlado por edad, sexo e ingreso familiar, se observa que una mayor tendencia al auto-ocultamiento se asocia a mayor presencia de sintomatología, $r=0,54, p<0,001$. Este resultado es consistente con la literatura, asociando al auto-ocultamiento con la presencia de sintomatología depresiva y ansiosa (e.g., Cepeda-Benito \& Short, 1998; Larson \& Chastain, 1990; Masuda et al., 2011; Uysal et al., 2010).

\section{Descriptivos de la EAO por Variables Sociodemográficas}

$\mathrm{Al}$ comparar los puntajes totales de la EAO por categoría muestral, sexo, edad e ingreso familiar (Tabla 4), se observa que los sujetos en tratamiento de salud mental tienden más al auto-ocultamiento que aquellos que no se encontraban en un tratamiento, siendo esta una diferencia pequeña, $t(186)=5,42, p<$ $0,001, d=0,28)$. En términos del sexo, hay una diferencia pequeña en la tendencia a auto-ocultar, con puntajes más altos para hombres, $t(190)=4,28, p<0,001, d=0,14$. Asimismo, a mayor edad mayor es el auto-ocultamiento, a su vez con una diferencia pequeña, $F(2,190)=9,22, p<0,001, \eta^{2}=0,01$. Y, por último, a menor ingreso familiar mayor es el auto-ocultamiento, con una diferencia mediana, $F(2,187)=6,09, p<$ $0,001, \eta^{2}=0,06$.

Sin embargo, al realizar una regresión lineal múltiple, las diferencias por categoría muestral, sexo y edad en la tendencia a auto-ocultar dejan de ser estadisticamente significativas (Tabla 5). 
Tabla 4

Descriptivos de la EAO e Intervalos de Confianza de las Medias por Categoría Muestral, Sexo, Edad e Ingreso Familiar Mensual

\begin{tabular}{|c|c|c|c|c|c|c|c|c|c|c|c|}
\hline \multirow{2}{*}{ Descriptivo } & \multicolumn{2}{|c|}{ Categoría muestral* } & \multicolumn{2}{|c|}{ Sexo } & \multicolumn{3}{|c|}{ Edad } & \multicolumn{3}{|c|}{ Ingreso familiar mensual } & \multirow{2}{*}{$\begin{array}{c}\text { Muestra } \\
\text { total }\end{array}$} \\
\hline & Clínica & $\begin{array}{c}\text { No } \\
\text { clínica }\end{array}$ & Masc. & Fem. & $\begin{array}{l}18 \text { a } 30 \\
\text { años }\end{array}$ & $\begin{array}{l}31 \text { a } 45 \\
\text { años }\end{array}$ & $\begin{array}{l}46 \text { años o } \\
\text { más }\end{array}$ & $<\mathrm{US} \$ 650$ & $\begin{array}{l}\text { US\$ } 650- \\
\text { US } \$ 1.650\end{array}$ & $\begin{array}{c}>\mathrm{US} \$ \\
1.650\end{array}$ & \\
\hline Promedio & 28,06 & 25,91 & 25,09 & 26,29 & 26,77 & 29,73 & 30,55 & 27,97 & 25,31 & 23,13 & 25,78 \\
\hline Desviación estándar & 6,65 & 8,58 & 8,14 & 8,68 & 9,06 & 8,52 & 9,52 & 9,51 & 6,71 & 7,64 & 8,58 \\
\hline Puntaje mínimo & 10 & 10 & 10 & 10 & 10 & 10 & 11 & 10 & 12 & 10 & 10 \\
\hline Puntaje máximo & 49 & 42 & 49 & 46 & 46 & 49 & 47 & 49 & 38 & 43 & 49 \\
\hline $\begin{array}{l}\text { Intervalo de confianza } \\
\text { límite inferior }\end{array}$ & 24,75 & 24,27 & 23,47 & 24,49 & 23,75 & 24,64 & 21,46 & 25,81 & 23,38 & 21,21 & 24,57 \\
\hline $\begin{array}{l}\text { Intervalo de confianza } \\
\text { límite superior }\end{array}$ & 31,37 & 26,94 & 26,71 & 28,09 & 26,54 & 30,27 & 32,40 & 30,13 & 27,24 & 25,04 & 26,99 \\
\hline$N$ & 18 & 170 & 100 & 92 & 137 & 42 & 14 & 77 & 49 & 64 & 193 \\
\hline
\end{tabular}

*Categoría muestral clínica corresponde a aquellos pacientes que dijeron estar actualmente en un tratamiento de salud mental y no clínica, a aquellos que reportaron no estar en un tratamiento de salud mental.

Tabla 5

Coeficientes del Modelo de Regresión Lineal

\begin{tabular}{lrcrrr}
\hline \multicolumn{1}{c}{ Modelo } & \multicolumn{1}{c}{$b$} & Error típico & \multicolumn{1}{c}{$B$} & \multicolumn{1}{c}{$t$} & \multicolumn{1}{c}{$p$} \\
\hline Constante & 11,190 & 1,790 & & 6,230 & $<0,001$ \\
Categoría muestral & $-1,010$ & 0,905 & $-0,050$ & $-1,120$ & 0,264 \\
Edad & $-0,003$ & 0,030 & $-0,005$ & $-0,106$ & 0,910 \\
Sexo & 0,720 & 0,750 & 0,030 & 0,970 & 0,333 \\
Ingreso familiar mensual & $-0,360$ & 0,140 & $-0,090$ & $-2,490$ & 0,010 \\
Puntaje total OQ & 0,360 & 0,030 & 0,520 & 11,980 & $<0,001$ \\
\hline
\end{tabular}

Nota. Variable dependiente: Puntaje total EAO; $b=$ coeficiente de regresión no estandarizado; $B=$ coeficiente de regresión estandarizado. 


\section{Discusión}

Según lo presentado en la revisión teórica y esta investigación, el concepto de auto-ocultamiento debe ser entendido como una predisposición a ocultar información personal percibida por el sujeto como negativa o estresante y que activamente decide no revelar. Un gran auto-ocultador es aquella persona que tiene muchos secretos negativos acerca de sí mismo y los oculta de manera repetida en el tiempo. El autoocultamiento no se refiere al acto de ocultar información asociado a circunstancias externas o a cierto tipo específico de información y no abarca el fenómeno de mantener un secreto en toda su amplitud. El presente trabajo tuvo por objetivo realizar una adaptación al español y revisar las propiedades psicométricas de la EAO de Larson y Chastain (1990), como instrumento de evaluación del fenómeno del auto-ocultamiento. Tener presente las limitaciones del concepto de auto-ocultamiento será relevante y determinante al elegir la escala como una medida a incluir en algún estudio, en la práctica clínica o al interpretar sus resultados.

Esta investigación, con una muestra de adultos chilenos, demuestra que la versión en español de la EAO presenta propiedades psicométricas adecuadas, satisfactorias y similares a las de la versión original con población adulta norteamericana (Larson \& Chastain, 1990). Respecto de la confiabilidad, se obtuvo una alta consistencia interna y una aceptable estabilidad a través del tiempo, lo que indica que esta escala es una herramienta confiable para ser utilizada en la medición de la tendencia a auto-ocultar.

Estos resultados también constituyen un apoyo a la validez de la escala, observándose una alta correlación entre presencia de sintomatología ansiosa y depresiva con la tendencia a auto-ocultar. Lo anterior es coherente con los hallazgos de Larson y Chastain (1990) e investigaciones posteriores y apoya la idea de que auto-ocultar se asocia a consecuencias dañinas para la salud psicológica (Wismeijer et al., 2008).

Con el propósito de que la EAO midiese el auto-ocultamiento en toda su extensión, la intención inicial de Larson y Chastain (1990) fue medir varias dimensiones, entendiendo que el fenómeno de ocultamiento es complejo por naturaleza (Kelly, 1999, Wismeijer et al., 2008). Sin embargo, los resultados de este estudio, así como las revisiones de la escala realizadas en Holanda (Wismeijer et al., 2008), Canadá (Cramer \& Barry, 1999) y Turquía (Deniz \& Çok, 2010), corroboran la unidimensionalidad de la escala. A partir de estos resultados, se puede inferir que, aunque la escala mide la posesión de al menos un secreto angustiante y la renuencia a compartirlo con otros, su estructura no representa la naturaleza multidimensional del auto-ocultamiento. Alternativamente, puede cuestionarse la multidimensionalidad teórica propuesta por Larson y Chastain (1990). Ambos aspectos deben ser evaluados en estudios posteriores.

A pesar de las grandes diferencias culturales entre las poblaciones norteamericana, turca y chilena, tanto las adaptaciones en turco como en español muestran coeficientes de confiabilidad, de validez y una estructura factorial muy similares entre sí y los de la escala original. Esto las hace equivalentes y comparables, configurándose como una fortaleza de la escala permitir estudios interculturales. Además, debido al gran número de hispanohablantes en el mundo, contar con una versión en español de la EAO favorece la comprensión del individuo y amplía su uso en múltiples temas y escenarios.

Debido a que la muestra en el estudio original de Larson y Chastain (1990) se basó en la autoselección y en una muestra sesgada hacia el sexo femenino (29 hombres, 277 mujeres), la generalización de los hallazgos acerca de la independencia del constructo respecto a factores sociodemográficos es limitada. En el presente estudio se utilizó una muestra diversa sociodemográficamente para que permitiera evaluar posibles diferencias por categoría muestral, sexo, edad e ingreso familiar mensual. Si bien existen diferencias por sexo, edad y categoría muestral, estas dejaron de ser significativas cuando se controló por ingreso familiar mensual y bienestar psicológico (OQ). Estos resultados no son concordantes con la literatura expuesta, que ha asociado un mayor auto-ocultamiento a las personas que estaban en un proceso psicoterapéutico (e.g., Cramer \& Barry, 1999; Kelly \& Achter, 1995) y mayor tendencia de los hombres a auto-ocultar (Cramer \& Barry, 1999). Estas diferencias entre las investigaciones podrían deberse a las diferencias culturales y sociales de las muestras, como un factor influyente en el fenómeno. No se encontraron en la literatura investigaciones que reportaran una diferencia a auto-ocultar según edad o ingreso familiar mensual.

Este estudio tuvo como limitación que la muestra elegida por conveniencia no es representativa de la población nacional. Además, no se incluyó una población clínica de manera intencionada, cuando el mayor uso de la EAO es probablemente en poblaciones clínicas. A su vez, los resultados del presente trabajo arrojan poca información acerca de las características personales o las variables que influyen o intervienen 
en la tendencia a auto-ocultar. Como criterio de validez de la adaptación de la EAO no se incluyó un análisis en donde se comparasen los resultados de la adaptación de la EAO con un instrumento que midiese el mismo constructo, ya que la EAO se configura como el único instrumento que mide el concepto de auto-ocultamiento sin otros fenómenos concurrentes. Si bien podría considerarse como criterio de validez el comparar la EAO con una escala que midiese el constructo de la auto-revelación, los investigadores han concluido que la ocultación y la revelación no son extremos opuestos de un continuo bipolar, ya que los procesos mentales implicados en ambos fenómenos son diferentes: el ocultamiento implica un proceso activo de inhibición, mientras que la revelación implica un proceso activo de enfrentamiento de la angustia. De este modo, una persona con una baja tendencia al auto-ocultamiento no significa que sea un gran auto-revelador de su angustia; simplemente puede ser alguien que pasivamente la ignore (Kahn \& Hessling, 2001).

Estudios futuros deberían centrarse en las características personales y contextuales que pueden afectar a la tendencia al auto-ocultamiento. Por ejemplo, sería útil estudiar el funcionamiento diferencial de cada ítem para identificar cómo se comportan dependiendo de la cultura, el género, la etnia, el nivel socioeconómico y el nivel educativo. Además, es necesario investigar sobre la relación entre autoocultamiento y psicoterapia. La EAO no identifica si los individuos auto-ocultan con todas las personas con las que interactúan o si hay excepciones, como su psicoterapeuta. Si se considera que aquellos que puntúan alto en alto-ocultamiento son 50\% más propensos a consultar a un terapeuta y, a su vez, mantienen actitudes menos favorables durante la terapia (Kelly \& Achter, 1995), se hace relevante estudiar qué efectos tiene el auto-ocultamiento en la alianza terapéutica, los resultados de la psicoterapia y la probabilidad de que el paciente abandone el tratamiento. A su vez, sería interesante investigar el efecto sobre la alianza, la adherencia y el resultado, si el terapeuta tuviera conocimiento de la tendencia del paciente al auto-ocultamiento. Contar con una versión en español de la EAO permitirá responder a estas y otras preguntas con pacientes de habla hispana, lo que tiene relevantes implicancias prácticas.

En resumen, este estudio muestra que la adaptación al español de la EAO es confiable, válida y con propiedades psicométricas muy similares a la versión original en inglés de Larson y Chastain (1990). Contar con una adaptación en español permitirá realizar estudios interculturales e investigaciones comparativas. Esta escala de autoaplicación es fácil de usar y será útil en contextos clínicos y de investigación.

\section{Referencias}

Bühl, A. (2011). SPSS 20. Introduction to modern data analysis. München, Alemania: Pearson.

Cepeda-Benito, A. \& Short, P. (1998). Self-concealment, avoidance of psychological services, and perceived likelihood of seeking professional help. Journal of Counseling Psychology, 45, 58-64. https://doi.org/10.1037/0022-0167.45.1.58

Corrigan, P. W. \& Rao, D. (2012). On the self-stigma of mental illness: Stages, disclosure, and strategies for change. The Canadian Journal of Psychiatry, 57, 464-469. https://doi.org/10.1177/070674371205700804

Cramer, K. M. \& Barry, J. E. (1999). Psychometric properties and confirmatory factor analysis of the Self-Concealment Scale. Personality and Individual Differences, 27, 629-637. https://doi.org/10.1016/S0191-8869(98)00222-0

Cruddas, S., Gilbert, P. \& McEwan, K. (2012). The relationship between self-concealment and disclosure, early experiences, attachment, and social comparison. International Journal of Cognitive Therapy, 5, 28-37. https://doi.org/10.1521/ijct.2012.5.1.28

Deniz, M. \& Çok, F. (2010). Psychometric properties and adaptation of the Self-Concealment Scale to the Turkish adolescents [Abstract y Summary]. Illköğretim Online (Elementary Education Online), 9, 424-432. Resumen extraído de http://ilkogretimonline.org.tr/index.php/io/article/view/1909/1745

Dorahy, M. J. \& Clearwater, K. (2012). Shame and guilt in men exposed to childhood sexual abuse: A qualitative investigation. Journal of Child Sexual Abuse, 21, 155-175. https://doi.org/10.1080/10538712.2012.659803

Errázuriz, P., Opazo, S., Behn, A., Silva, O. \& Gloger, S. (2017). Spanish adaptation and validation of the Outcome Questionnaire OQ30.2. Frontiers in Psychology, 8, Artículo 673. https://doi.org/10.3389/fpsyg.2017.00673

Farber, B. A., Berano, K. C. \& Capobianco, J. A. (2004). Clients' perceptions of the process and consequences of self-disclosure in psychotherapy. Journal of Counseling Psychology, 51, 340-346. https://doi.org/10.1037/0022-0167.51.3.340

Fivush, R. (2010). Speaking silence: The social construction of silence in autobiographical and cultural narratives. Memory, 18 , 88-98. https://doi.org/10.1080/09658210903029404

Hill, C. E., Gelso, C. J. \& Mohr, J. J. (2000). Client concealment and self-presentation in therapy: Comment on Kelly (2000). Psychological Bulletin, 126, 495-500. https://doi.org/10.1037/0033-2909.126.4.495

Hu, L.-t., \& Bentler, P. M. (1999). Cutoff criteria for fit indexes in covariance structure analysis: Conventional criteria versus new alternatives. Structural Equation Modeling, 6(1), 1-55. https://doi.org/10.1080/10705519909540118

Ironson, G., O'Cleirigh, C., Leserman, J., Stuetzle, R., Fordiani, J., Fletcher, M. \& Schneiderman, N. (2013). Gender-specific effects of an augmented written emotional disclosure intervention on posttraumatic, depressive, and HIV-disease-related outcomes: A randomized, controlled trial. Journal of Consulting and Clinical Psychology, 81, 284-298. https://doi.org/10.1037/a0030814

Kahn, J. H. \& Hessling, R. M. (2001). Measuring the tendency to conceal versus disclose psychological distress. Journal of Social and Clinical Psychology, 20, 41-65. https://doi.org/10.1521/jscp.20.1.41.22254 
Kelley, J. E., Lumley, M. A. \& Leisen, J. C. C. (1997). Health effects of emotional disclosure in rheumatoid arthritis patients. Health Psychology, 16, 331-340. https://doi.org/10.1037/0278-6133.16.4.331

Kelly, A. E. (1999). Revealing personal secrets. Current Directions in Psychological Science, 8, 105-109. https://doi.org/10.1111/1467-8721.00025

Kelly, A. E. \& Achter, J. A. (1995). Self-concealment and attitudes toward counseling in university students. Journal of Counseling Psychology, 42, 40-46. https://doi.org/10.1037/0022-0167.42.1.40

Kelly, A. E. \& Yip, J. J. (2006). Is keeping a secret or being a secretive person linked to psychological symptoms? Journal of Personality, 74, 1349-1370. https://doi.org/10.1111/j.1467-6494.2006.00413.x

Kline, R. B. (2016). Methodology in the social sciences. Principles and practice of structural equation modeling (4a ed.). Guilford Press.

Laird, R. D., Marrero, M. D., Melching, J. A. \& Kuhn, E. S. (2013). Information management strategies in early adolescence: Developmental change in use and transactional associations with psychological adjustment. Developmental Psychology, 49, 928937. https://doi.org/10.1037/a0028845

Lambert, M. J., Finch, A. M., Okiishi, J. \& Burlingame, G. M. (2005). Administration and scoring manual for the OQ-30.2. Salt Lake City, UT: OQMeasures.

Larson, D. G. (s.f.). Self-Concealment Research Collaborative [Blog]. Santa Clara, CA: Autor. Extraído de http://blogs.scu.edu/selfconcealment/

Larson, D. G. \& Chastain, R. L. (1990). Self-concealment: Conceptualization, measurement, and health implications. Journal of Social and Clinical Psychology, 9, 439-455. https://doi.org/10.1521/jscp.1990.9.4.439

Larson, D. G., Chastain, R. L., Hoyt, W. T. \& Aysenberg, R. (2015). Self-concealment: Integrative review and working model. Journal of Social and Clinical Psychology, 34, 705-e774. https://doi.org/10.1521/jscp.2015.34.8.705

Maas, J., Wismeijer, A. A. J., Van Assen, M. A. L. M. \& Aquarius, A. E. A. M. (2012). Is it bad to have secrets? Cognitive preoccupation as a toxic element of secrecy. International Journal of Clinical and Health Psychology, 12, 23-37. Extraído de https://www.researchgate.net/publication/259738675_Is_it_bad_to_have_secrets_Cognitive_preoccupation_as_a_toxic_element_of_secrecy

Masuda, A., Anderson, P. L., Wendell, J. W., Chou, Y -Y., Price, M., \& Feinstein, A. B. (2011). Psychological flexibility mediates the relations between self-concealment and negative psychological outcomes. Personality and Individual Differences, 50, $243-247$. https://doi.org/10.1016/j.paid.2010.09.037

Miller, L. C., Berg, J. H. \& Archer, R. L. (1983). Openers: Individuals who elicit intimate self-disclosure. Journal of Personality and Social Psychology, 44, 1234-1244. https://doi.org/10.1037/0022-3514.44.6.1234

Mokken, R. J. \& Lewis, C. (1982). A nonparametric approach to the analysis of dichotomous item responses. Applied Psychological Measurement, 6, 417-430. https://doi.org/10.1177/014662168200600404

Morales Vallejo, P. (2008). Estadística aplicada a las ciencias sociales. Madrid, España: Universidad Pontificia Comillas.

Moreno Fernández, F. \& Otero Roth, J. (2006). Demografía de la lengua española. Madrid, España: Instituto Complutense de Estudios Internacionales/Fundación Telefónica.

Nam, S. K., Choi, S. I., Lee, J. H., Lee, M. K., Kim, A. R. \& Lee, S. M. (2013). Psychological factors in college students' attitudes toward seeking professional psychological help: A meta-analysis. Professional Psychology: Research and Practice, 44, 37-45. https://doi.org/10.1037/a0029562

Pardo Merino, A. \& Ruiz Díaz, M. (2002). SPSS 11: guía para el análisis de datos. Madrid, España: McGraw-Hill.

Pennebaker, J. W., Colder, M. \& Sharp, L. K. (1990). Accelerating the coping process. Journal of Personality and Social Psychology, 58, 528-537. https://doi.org/10.1037/0022-3514.58.3.528

Pennebaker, J. W. \& O'Heeron, R. C. (1984). Confiding in others and illness rate among spouses of suicide and accidental-death victims. Journal of Abnormal Psychology, 93, 473-476. https://doi.org/10.1037/0021-843X.93.4.473

R Core Team (2017). R: A language and environment for statistical computing. Wien, Austria: R Foundation for Statistical Computing.

Rosseel, Y. (2012). Lavaan: An R package for structural equation modeling. Journal of Statistical Software, 48(2), 1-36. https://doi.org/10.18637/jss.v48/i02/

Schrimshaw, E. W., Siegel, K., Downing Jr., M. J. \& Parsons, J. T. (2013). Disclosure and concealment of sexual orientation and the mental health of non-gay-identified, behaviorally bisexual men. Journal of Consulting and Clinical Psychology, 81, 141-153. https://doi.org/10.1037/a0031272

Tabachnick, B. G. \& Fidell, L. S. (2001). Using multivariate statistics (4ª ed.). Boston, MA: Allyn \& Bacon.

Uysal, A., Lee Lin, H. \& Knee, C. R. (2010). The role of need satisfaction in self-concealment and well-being. Personality and Social Psychology Bulletin, 36, 187-199. https://doi.org/10.1177/0146167209354518

Wegner, D. M. \& Lane, J. D. (1995). From secrecy to psychopathology. En J. W. Pennebaker (Ed.), Emotion, disclosure \& health (pp. 25-46). Washington, DC: American Psychological Association.

Wheat, K., Brohan, E., Henderson, C. \& Thornicroft, G. (2010). Mental illness and the workplace: Conceal or reveal? Journal of the Royal Society of Medicine, 103, 83-86. https://doi.org/10.1258/jrsm.2009.090317

White, M. \& Epston, D. (1990/2002). Medios narrativos para fines terapéuticos (O. Castillo, M. Beyebach \& C. Sánchez, Trads.; Título original: Narrative means to therapeutic ends). Barcelona, España: Paidós.

Wismeijer, A. A. J. (2011). Self-concealers: Do they conceal what we always assumed they do? Personality and Individual Differences, 51, 1039-1043. https://doi.org/10.1016/j.paid.2011.08.019

Wismeijer, A. A. J., Sijtsma, K., Van Assen, M. A. L. M. \& Vingerhoets, A. J. J. M. (2008). A comparative study of the dimensionality of the Self-Concealment Scale using principal components analysis and Mokken scale analysis. Journal of Personality Assessment, 90, 323-334. https://doi.org/10.1080/00223890802107875

Wismeijer, A. A. J., Van Assen, M. A. L. M., Sijtsma, K. \& Vingerhoets, A. J. J. M. (2009). Is the negative association between selfconcealment and subjective well-being mediated by mood awareness? Journal of Social and Clinical Psychology, 28, 728-748. https://doi.org/10.1521/jscp.2009.28.6.728

Zhang, B., Wang, M., Li, J., Yu, G. \& Bi, Y. -L. (2011). The effects of concealing academic achievement information on adolescents' selfconcept. The Psychological Record, 61, 21-40. https://doi.org/10.1007/BF03395744

Fecha de recepción: Junio de 2017.

Fecha de aceptación: Junio de 2018. 